\title{
Comparison of ANB Angle, Yen Angle and W Angle in Chinese Population
}

\author{
Dr Surendra Maharjan,' Dr Chen Lili² \\ 'Lecturer, People's Dental College and Hospital, Kathmandu, Nepal \\ 2Professor, Tongji Medical College, Wuhan, China
}

Correspondence: Dr Surendra Maharjan; Email: maharjan.surendra@gmail.com

\section{ABSTRACT}

Introduction: An accurate, reliable and stable method of accessing sagittal jaw relationship with cephalometric analysis is essential in orthodontic diagnosis and treatment planning. Many methods have been developed to assess antero-posterior jaw discrepancy but they all have some shortcomings including ANB angle. Thus the purpose of this study is 1) To provide mean values and standard deviations for ANB angle, Yen angle and W angle for a sample of skeletal Class I, II and III Chinese males and females malocclusion patients; 2) To verify the existence of sexual dimorphism; 3) To compare these three methods for assessing sagittal jaw relationship 4) To assess the correlation between these and 5) To find out which is the most reliable amongst them.

Materials \& Method: 120 pretreatment lateral cephalograms of Chinese patients, including both males and females between 18 to 25 years old, were collected from the Department of Orthodontics; Wuhan Union Hospital which were divided into 3 groups as Class I, II and III skeletal pattern. Each pattern consists of 40 samples. The values of the males and females were compared with independent t-test while the inter-group comparisons were conducted with ANOVA and Tukey's test. Pearson correlation test was performed to correlate between these angles.

Result: There were no statistically significant differences between the mean values of these angles in male and female within the groups in all three classes. ANOVA analysis showed significant differences were found in ANB angle, Yen angle and W-angle in all the three Groups. Strong correlation existed between Yen angle and W angle.

Conclusion: There is no existence of sexual dimorphism in our study. ANB angle is affected by growth of Nasion while Yen angle is affected by rotation of jaws. ANB angle is easy to assess while W angle is most stable and reliable.

Keywords: ANB angle, Sagittal discrepancy, W angle, Yen angle.

\section{INTRODUCTION}

In orthodontics, the cephalometer was introduced in 1931 by Broadbent in USA ${ }^{1}$ and Hofrath in Germany ${ }^{2}$ independently. Initially it was developed as a device to study craniofacial growth and development. Later it was also used for growth prediction, diagnosis and treatment planning and assessment of treatment progress of the patient.

During diagnosis and treatment planning of skeletal malocclusions, proper evaluation of sagittal jaw relationship is an important step which is generally determined by cephalometric analysis. Since Wylie's (1947) ${ }^{3}$ first attempt to describe AP jaw relationship, many other cephalometric parameters have been proposed. Downs $(1948)^{4}$ was the first to evaluate anteroposterior jaw relationship cephalometrically with introduction of points $A$ and $B$ constructing $A B$ plane angle. After few years, in 1952 Riedel $^{5}$ introduced ANB angle which became one of the most important measurement in many analyses since that time e.g. Steiner's analyses. ${ }^{6}$

Any cephalometric analysis based on either angular or linear measurements has obvious shortcomings and limitations, which have been discussed in detail by Moyers et $\mathrm{al}^{7}$ and by Appasaheb Naragond et $\mathrm{al}^{8}$ Although the ANB angle is still very popular, useful and easy to analyze, some authors have shown that point $A$ and $B$ are influenced by growth and dentoalveolar remodeling during orthodontic treatment. Also the position of nasion is not fixed during growth, and any displacement of nasion will directly affect the ANB angle. There is often a difference between the interpretation of this angle and the actual discrepancy between the apical bases. It is also noted that rotation of the jaws by either growth or orthodontic treatment 


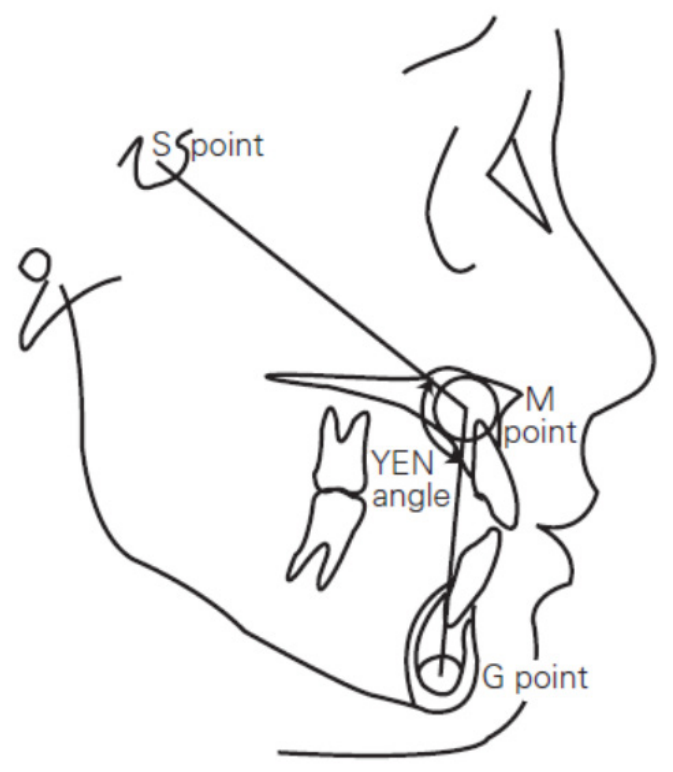

Figure 1: Yen Angle

can also change the value of ANB reading. ${ }^{10}$

Because of these problem, Neela et al (2009) introduced a new sagittal dysplasia indicator called YEN angle ${ }^{11}$ (Figure 1). It uses three skeletal landmarks point $S$ (midpoint of the sella turcica), point $M$ (midpoint of the premaxilla), and point $G$ (centre of the largest circle that is tangent to the internal inferior, anterior, and posterior surfaces of the mandibular symphysis).

When point S, M and $G$ connected, they form Yen angle which is measured at $M$.

Since it measures an angle between line SM and MG, rotation of jaw because of growth or orthodontic treatment can mask true basal dysplasia, similar to ANB angle. ${ }^{12}$

To overcome these existing problems, a measurement was developed and named the $W$ angle by Bhad et al at $(2011)^{12}$ ( Figure 2). It is a new measurement for assessing the skeletal discrepancy between the maxilla and the mandible in the sagittal plane. It also uses the same landmarks used in Yen angle - point $S$, point $M$, and point $G$ but with different angle, the angle between the perpendicular line from point $M$ to $S-G$ line and the $M-G$ line.

So the purpose of this study is

1. To provide mean values and standard deviations for Yen angle, and $\mathrm{W}$ angle for a sample of skeletal Class I, II and III Chinese males and females;

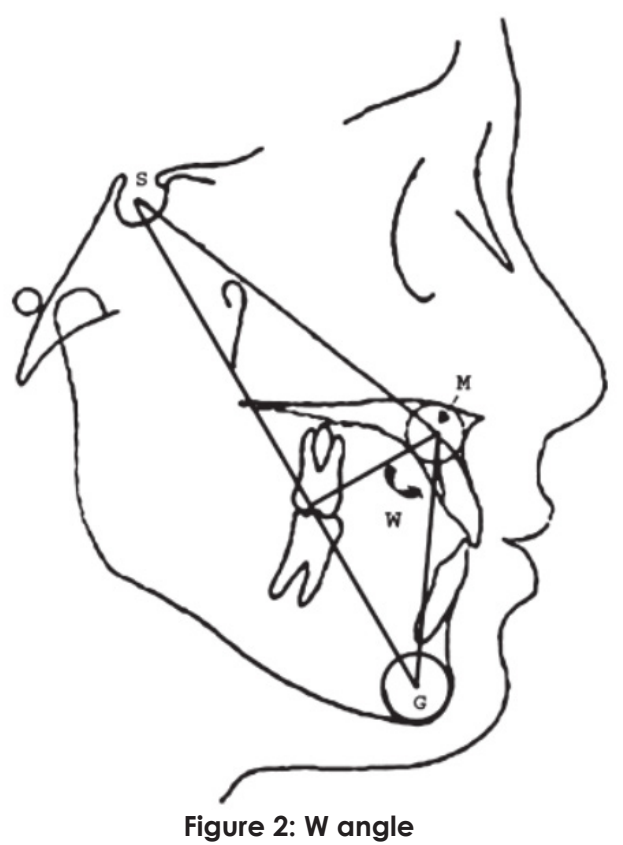

2. To verify the existence of sexual dimorphism;

3. To compare these three methods of cephalometric analysis for assessing sagittal jaw relationship with one another;

4. To assess the correlation between these angles with each other in different classes;

5. To find out which is the most reliable amongst them.

\section{MATERIALS AND METHOD}

For our study, the pretreatment lateral cephalograms of Chinese patients, including both males and females, were collected from the Department of Orthodontics; Wuhan Union Hospital affiliated to Huazhong University of Science and Technology. Only the radiographs with acceptable radiographic quality and minimal radiographic distortion were considered for the study. The radiographs were available as digital images which were printed in photo and were traced. It consisted of 120 pretreatment lateral cephalograms of 18 to 25 year old individuals who had never undergone orthodontic treatment. They were divided into 3 groups

Group I - Class I skeletal pattern group ( $\mathrm{n}=40,18$ male; 22 female)

Group II - Class II skeletal pattern group ( $n=40,15$ male; 25 female)

Group III - Class III skeletal pattern group (n=40, 19 male; 21 female) 
The following inclusion criteria was taken:

1. For the Class I skeletal pattern group- ANB angle of $1^{\circ}$ to $3^{\circ}$, Wits appraisal between 0 and $-3 \mathrm{~mm}$ and Pleasant profile.

2. For the Class II skeletal pattern group- ANB angle was above $4^{\circ}$, Wits appraisal greater than or equal to $-1 \mathrm{~mm}$ and the profile had a Class II appearance.

3. For the Class III skeletal pattern group- ANB angle was less than or equal to $1^{\circ}$, Wits appraisal less than or equal to $-4 \mathrm{~mm}$ and the profile had a Class III appearance.

The mean, standard deviation and $\mathrm{P}$ value were calculated for each parameter.

Comparison between both genders is done by using Independent sample t-test. The oneway analysis of variance (ANOVA) was used followed by posthoc Tukey's test to determine whether there were statistically significant differences between mean values of all the three groups. Pearson's correlation coefficient also used to study the correlation between these groups. All statistics were performed in IBM SPSS Statistics (2014).

\section{RESULT}

Independent $t$ tests showed that there were no statistically significant differences between the mean values of these angles of male and female sexes within the groups since $p$ value is greater than 0.05 . Therefore, all measurements belonging to both sexes were combined in each group and were considered for analysis.

Table 1 shows the descriptive and comparative statistics including mean, standard deviation, and coefficient of variation for the total sample. It also shows the comparison between the three skeletal relation using one way ANOVA followed by Tukey's HSD test to show the statistical significance between each two skeletal pattern (Table 2). These statistics show that mean values of each classes in each group are statistically significant and thus are different form each other since $p$ value is less than 0.05. Table 3 shows the correlation between these angles using Pearson's correlation coefficient. It shows that significant correlation between Yen angle and $W$ angle in all three classes.

Table 1: Descriptive and comparative statistics among the three skeletal relations for the total sample

\begin{tabular}{|c|c|c|c|c|c|c|}
\hline Variable & Class & No. & Mean & S.D. & F test & Sig. \\
\hline \multirow{3}{*}{ ANB } & 1 & 40 & 2.1600 & 0.893 & \multirow{3}{*}{199.403} & \multirow{3}{*}{0.000} \\
\hline & $\|$ & 40 & 5.7125 & 1.390 & & \\
\hline & III & 40 & -1.1250 & 2.074 & & \\
\hline \multirow{3}{*}{ Yen } & I & 40 & 124.9500 & 2.68137 & \multirow{3}{*}{216.420} & \multirow{3}{*}{0.000} \\
\hline & $\|$ & 40 & 119.7500 & 1.88108 & & \\
\hline & III & 40 & 130.7875 & 2.48557 & & \\
\hline \multirow{3}{*}{ W } & 1 & 40 & 55.8125 & 2.00220 & \multirow{3}{*}{206.695} & \multirow{3}{*}{0.000} \\
\hline & $\|$ & 40 & 51.4500 & 1.71644 & & \\
\hline & III & 40 & 61.7625 & 2.93299 & & \\
\hline
\end{tabular}

The means are significant at the 0.05 level.

Table 2: Comparison between each two different skeletal pattern using TUKEY HSD

\begin{tabular}{|c|c|c|c|c|c|c|}
\hline \multirow[t]{2}{*}{ Variable } & \multicolumn{2}{|c|}{ Class I-class II } & \multicolumn{2}{|c|}{ Class II-class III } & \multicolumn{2}{|c|}{ Class I-class III } \\
\hline & Mean & Sig. & Mean & Sig. & Mean & Sig. \\
\hline ANB & $-3.55250^{*}$ & 0.000 & $6.83750 *$ & 0.000 & $3.28500^{*}$ & 0.000 \\
\hline Yen & $5.20000 *$ & 0.000 & $-11.03750 *$ & 0.000 & $-5.83750 *$ & 0.000 \\
\hline W & $4.36250 *$ & 0.000 & $-10.31250 *$ & 0.000 & $5.95000 *$ & 0.000 \\
\hline \multirow{3}{*}{ W } & 1 & 40 & 55.8125 & 2.00220 & \multirow{3}{*}{206.695} & \multirow{3}{*}{0.000} \\
\hline & ॥ & 40 & 51.4500 & 1.71644 & & \\
\hline & III & 40 & 61.7625 & 2.93299 & & \\
\hline
\end{tabular}

\footnotetext{
*. The mean difference is significant at the 0.05 level.
} 
Table 3: The correlation between the variables in class I, II and III skeletal

\begin{tabular}{|c|c|c|c|}
\hline Variables & Class & W & Yen \\
\hline \multirow{3}{*}{ ANB } & I & -0.234 & 0.012 \\
\cline { 2 - 4 } & $\|$ & -0.079 & -0.271 \\
\cline { 2 - 4 } & III & $-0.494^{*}$ & -0.236 \\
\hline \multirow{3}{*}{ Yen } & I & $0.746^{*}$ & \\
\cline { 2 - 4 } & II & $0.425^{*}$ & \\
\cline { 2 - 4 } & III & $0.714^{*}$ & \\
\hline
\end{tabular}

*. Correlation is significant at the 0.01 level.

\section{DISCUSSION}

Cephalometric radiograph is a valuable tool in orthodontic diagnosis and treatment planning. Even before Angle introduced his classification of malocclusion, the anteroposterior relationship of mandible to maxilla was the most important diagnostic criterion. Assessing this sagittal relationship is a challenging issue in orthodontics. Also the clinicians with increasing frequency are treating malocclusions in conjunction with orthognathic surgery. Thus a method of maxillomandibular assessment that provides accurate data on this relationship at an early age would be highly desirable. ${ }^{13}$

Since its introduction in 1952, ANB angle is still widely used and popular but many authors have shown that the point $N$ is not fixed and any displacement will directly affect the ANB angle. ${ }^{14}$ Other factors like age of the patient, change of the spatial position of the nasion either in the vertical or anteroposterior direction or both, upward or downward rotation of the SN plane, upward or downward rotation of the jaws, change in the angle SN to the occlusal plane and the degree of facial prognathism also affect the ANB angle. ${ }^{15,16}$ So many other new cephalometric measurements have been introduced claiming more accurate, easy to trace and less affected by the various factors.

In our study, the mean value for Yen angle in the Class | skeletal pattern group was $124.95^{\circ} \pm 2.68^{\circ}$, for Class || $119.75^{\circ} \pm 1.88^{\circ}$ and for Class III $130.78^{\circ} \pm 2.48^{\circ}$. The mean value of our present study is higher than the result of Neela et al." Similarly YEN angle norm for Indian population was $113.733 \pm 1.16,{ }^{17}$ for Pakistani population was found to be $119.5 \pm 3$, and for the Bangladeshi population it was $120.5 \pm 3 .{ }^{18}$

The main advantage of Yen angle is that it eliminates the difficulty in locating points $A$ and $B$, or the functional occlusal plane used in Wits analyses. As it is not influenced by growth changes, it can be used in mixed dentition as well. But since it measures an angle between line SM and MG, rotation of jaw because of growth or orthodontic treatment can mask true basal dysplasia, similar to ANB angle.

In our study, the mean value for $\mathrm{W}$ angle in the Class | skeletal pattern group was $55.81^{\circ} \pm 2.00^{\circ}$, for Class || $51.45 \pm 1.71^{\circ}$ and for Class III $61.76^{\circ} \pm 2.93^{\circ}$. The mean value of our present study is slightly higher than the result of Wasundhara et al. ${ }^{12}$ Similarly our values are slightly higher than the Pakistani population in class I and II while almost same in class III as research done by Hana Pervez and Imtiaz Ahmed. ${ }^{19}$ Also the values for $W$ angle was established for the Pakistani $54.5 \pm$ 3 for Bangladeshi population $55 \pm 324$ and for Indian population $52.866 \pm 1.30 .^{17}$

W angle also uses the same landmarks used in Yen angle but with different angle, the angle between the perpendicular line from point $M$ to $S-G$ line and the $M-G$ line. The authors claim that $W$ angle reflects true sagittal dysplasia not affected by growth rotations. ${ }^{12}$ The geometry of the $\mathrm{W}$ angle gives it the advantage to remain relatively stable even when the jaws are rotated or growing vertically. This is a result of rotation of the S-G line along with jaw rotation, which carries the perpendicular from point $M$ with it. Because the $M-G$ line is also rotating in the same direction, the $W$ angle remains relatively stable. So this angle can also be used for evaluation of treatment progress because it reflects true changes of the sagittal relationship of the jaws either due to growth or due to the orthodontic or orthognathic treatment. However, precisely tracing the premaxilla and locating its centre is not always easy. To accurately use this angle, the cephalometric $x$-rays must be high quality. It is then much easier for the clinician to follow the contour of premaxilla and locate its centre. 
A highly significant positive correlation existed between $W$ angle and Yen angle whereas weak negative correlation to ANB angle in all three classes. The strong correlation between the $\mathrm{W}$ angle and Yen angle is due to the fact that both of them share the common landmarks. From the experiment done by $D$ Veeranarayana et al. $^{20}$ also showed that there is significant correlation between $W$ angle and Yen angle.

Cranial base length (position of point N) can sometimes camouflage true skeletal classes I, II, and III patterns. In this regard, W angle can be a valuable tool for planning orthopaedic or orthognathic procedures as this angle is independent of cranial base length and remains relatively stable during growth and orthodontic or orthognathic treatment. But in Class II and Class III skeletal cases, W angle cannot determine which jaw is prognathic or retrognathic. In such cases ANB gives idea about the position of jaws however both the angle depends upon point N. So cephalometric analyses based on angular or linear measurements have obvious limitations and hence dependency on only one parameter for skeletal assessment is discouraged.

\section{CONCLUSION}

Due to the large variability in human population, a single cephalometric analysis may not provide an accurate diagnosis. Moreover, cephalometric is not an exact science and the various analyses based on angular and linear parameters have obvious limitations. Hence, it is imperative that a clinician be aware of a range of cephalometric analyses to be used appropriately as the need arises.

So from our study, we can conclude that there is no significant difference between mean values for male and female. The mean value of Yen angle for class I, II and III are 124.95, 119.75 and 130.78 while that for $W$ angle are 55.81, 51.45 and 61.76 . Construction and interpretation of ANB angle is easy to use clinically but can be affected by the growth of Nasion while Yen angle and $W$ angle have eliminated the difficulties in locating points A, B and N. Yen angle is affected by rotation of jaws while $\mathrm{W}$ angle remains stable. $\mathrm{W}$ angle is thus more reliable having only shortcoming that it cannot determine which jaw is prognathic or retrognathic.

\section{REFERENCES}

1. Broadbent BH. A new X-ray technique \& its application to orthodontia. . Angle Orthodontist 1931;1:45-66.

2. Hofrath H. Bedeutung der Rontgenfern und Abstands Aufnahme fur die Diagnostic der Kieferanomalein. Fortschr der Orthod. $1931 ; 1: 231-58$.

3. Wylie WL. The assessment of anteroposterior dysplasia. Angle Orthod. 1947;17:97-109.

4. Downs WH. Variations in facial relationship: their significance in treatment and prognosis. Am J Orthod 1948;34:812-40.

5. Riedel RA. The relation of maxillary structures to cranium in malocclusion and in normal occlusion. Angle Orthod. 1952;22:142-5.

6. Steiner CC. Cephalometrics for you and me. Am J Orthod. 1953;39:729-55.

7. Moyers RE, Bookstein FL, Guire KE. The concept of pattern in craniofacial growth. Am J Orthod. 1979;76:136-48.

8. Appasaheb N, Smitha K, Roshan SN, Sathish KS. Diagnostic Limitations of Cephalometrics in Orthodontics. A Review Journal of Dental and Medical Sciences. 2012;3(1).

9. Baik CY, Ververidou M. A new approach of assessing sagittal discrepancy: The Beta angle. Am J Orthod. 2004;126:100-5.

10. Jacobson A. The 'Wits' appraisal of jaw disharmony. Am J Orthod. 1975;67:125-38.

11. Neela PK, Mascarenhas R, Husain A. A new sagittal dysplasia indicator: the YEN angle. World J Orthod. 2009;10(2):147-51.

12. Bhad WA, Nayak S, Doshi UH. A new approach of assessing sagittal dysplasia: the W angle. European journal of orthodontics. Feb 2013;35(1):66-70.

13. Nanda RS, Merrill RM. Cephalometric assessment of sagittal relationship between maxilla and mandible. Am J Orthod. 1994;105(4):328-44.

14. Freeman RS. Adjusting A-N-B angles to reflect the effect of maxillary position. Angle Orthod. 1981;51 (2):162-71.

15. Chang HP. Assessment of anteroposterior jaw relationship. Am J Orthod Dentofacial Ortho. 1987;92(2):117-22.

16. Sara MA-M. The relation between $\mathrm{W}$ angle and other methods used to assess the sagittal jaw relationship. J Bagh College Dentistry. 2012;24(2):144-9.

17. Kavita S, Anil S, Vivek M, Jaj HS, Vishal S, Madhurima N. Comparison of Different Angular Measurements to Assess Sagittal Skeletal Discrepancy - A Cephalometric Study. Indian Journal of Dental Sciences. 2012;4(2).

18. Mohammad Khursheed Alam et al. Validity of W Angle and YEN Angle in a Sample from Pakistani and Bangladeshi Populations. JHard Tissue Biology. 2014;23(3):351-6.

19. Pervez H, Ahmed I. A new cephalometric tool W-angle for the evluation of anteroposterior skeletal discrepancy in orthodontic patients. Int J Dent Health Sci. 2014;1 (3):299-304.

20. D. Veeranarayana et al. Reliability, Accuracy and Interchangeability of Different Cephalometric Methods for Assessing Sagittal Jaw Relationships. J Res Adv Dent. 2014;3(1):232-8. 